\title{
Immersion in Water During Active Labor Decreases Postpartum Hematocrit Fall Following Vaginal Delivery
}

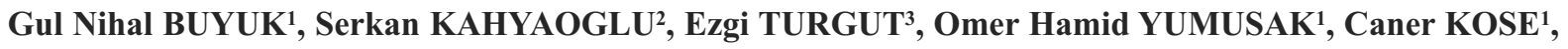 \\ Yaprak USTUN ${ }^{1}$
}

Ankara, Turkey

\begin{abstract}
OBJECTIVE: The objective of the study was to investigate the effect of immersion in water strategy during labor on postpartum bleeding by calculating the postpartum reduction rates of the hematocrit values of the patients.

STUDY DESIGN: The study groups consisted of 84 women undergoing vaginal delivery with immersion in water during labor (group 1) and the control group (group 2) of 84 women undergoing normal vaginal delivery. Patients who have received additional medical and surgical interventions for the alleviation of postpartum hemorrhage, patients who have undergone an episiotomy and/or perineal trauma were not included in the study. All data were taken from patients who have delivered with spontaneous vaginal delivery. Postpartum hematocrit fall rates of the groups have been compared and the effect of immersion in water on postpartum hemorrhage has been evaluated.
\end{abstract}

RESULTS: The study groups consisted of 84 women undergoing vaginal delivery with immersion in water during labor (Group 1) and the control group (Group 2) of 84 women undergoing vaginal delivery at the hospital. The women in the two groups were matched with respect to age, parity, birth weight and gestational age. The mean age of the women was $29.8 \pm 4.8$ and $30.5 \pm 4.9$ respectively. The mean hematocrit difference in the first group was $2.08 \pm 1.88$ and in the second group was $3.81 \pm 1.55$. The mean percentage of hematocrit reduction in the first group was $5.71 \%$ and in the second group $10.23 \%$.

CONCLUSION: Our data showed that mean hematocrit level decreases among women following vaginal delivery more than women who give birth vaginally within immersion in water during labor. The percentage of hematocrit reduction in the water birth group was lower than in the control group. Water birth seems to facilitate uterine contractions more efficiently following vaginal delivery.

Keywords: Hematocrit, Postpartum hemorrhage, Vaginal delivery, Water birth

Gynecol Obstet Reprod Med 2019;25(1):18-21

${ }^{1}$ University of Health Sciences, Zekai Tahir Burak Women Health Care, Education and Research Hospital Department of Obstetrics and Gynecology, and ${ }^{2}$ Department of Reproductive Endocrinology, Ankara Turkey

${ }^{3}$ Gazi University, Faculty of Medicine Department of Perinatology, Ankara, Turkey

Address of Correspondence: Gul Nihal Buyuk

University of Health Sciences, Zekai

Tahir Burak Women Health Care,

Education and Research Hospital, 06230 Altindag, Ankara, Turkey gnu@windowslive.com

Submitted for Publication 24.07.2018

Accepted for Publication:

01.10 .2018

ORCID IDs of the authors:

GN.B.: 0000-0003-4405-2876, S.K.: 0000-0001-8964-3552,

E.T.: 0000-0002-5509-7888, OH.Y.: 0000-0002-6632-5890,

C.K.: 0000-0002-3044-4804, Y.U.: 0000-0002-1011-3848

\begin{tabular}{|c|c|}
\hline Quick Response Code: & Access this article online \\
\cline { 2 - 2 } & $\begin{array}{l}\text { Website: www.gorm.com.tr } \\
\text { e- mail: info@gorm.com.tr }\end{array}$ \\
\cline { 2 - 2 } & DOI:10.201613/GORM.2018.824 \\
\hline
\end{tabular}

How to cite this article: Buyuk GN. Kahyaoglu S. Turgut E. Yumusak OH. Kose C. Yaprak U. Immersion in Water During Active Labor Decreases Postpartum Hematocrit Fall Following Vaginal Delivery. Gynecol Obstet Reprod Med 2019;25(1):18-21

\section{Introduction}

Water birth is an option for birth all over the world. Immersion in water during labor has been popularized over the past several decades. Carefully managed water birth is both an attractive and low-risk birth management for healthy pregnancies (1).

The prevalence of this practice is uncertain because it has not yet been studied in births outside of homes and birth centers, and the data are not recorded on birth certificates (1). For example, the United Kingdom has recently reported the rates of immersion ranging from $1.5 \%$ of hospital deliveries to $58 \%$ of births in freestanding midwifery units (2).

Commonly, immersion is referred to as "water birth," but effects and outcomes of immersion may be different during the first and the second stages of labor, including delivery. ACOG draws a distinction between these two events and declared and opinion that "immersion in water during the first stage of labor may be associated with decreased pain or the use of anesthesia and a decreased labor duration." A warning has also been stated that there are no known benefits for ei- 
ther mother or baby during the second stage of labor and cause concern for serious harm $(3,4)$. Immersion in water during the first stage of labor may be associated with shorter labor and decreased use of spinal and epidural analgesia and may be offered to healthy women with uncomplicated pregnancies between 37 0/7 weeks and $416 / 7$ weeks of gestation. The recommendation of the American College of Obstetricians and Gynecologists is that birth occurs on land, not in water (4-10).

In addition to providing freedom of movement and comfort to the mother in the water, the water lift increases the effectiveness of contractions by reducing the pressure on the uterus, thus shortening the duration of the action. Keeping the water temperature around the normal body temperature helps the mother by providing endorphin release (5-8). It also reduces postnatal breastfeeding problems as it provides pain relief for the mother $(6,8)$. Water birth increases their chances of attaining the goal of a natural birth without intervention and continues to provide a platform for maternity care reform, discoveries about consciousness and birth, and a new respect for fetal and newborn development (4).

Immersion during labor and delivery needs to establish rigorous protocols for candidate selection; maintenance and cleaning of tubs and pools; infection control procedures, including standard precautions and personal protective equipment for health care personnel; monitoring of women and fetuses at appropriate intervals while immersed, and moving women from tubs if urgent maternal or fetal concerns or complications develop (4).

The water birth cabin used in our hospital is ovoid, $161 \mathrm{~cm}$ in length, $124 \mathrm{~cm}$ in width and $65 \mathrm{~cm}$ in depth. Before each application, culture is taken to check that the cuvette does not contain an infectious agent. The bath is filled with filtered water at $34-37{ }^{\circ} \mathrm{C}$. After each birth, the bathtub is cleaned and filled with water again and circulated with $5.000 \mathrm{ppm}$ chlorine tablet. Cultures are removed after cleaning. After making sure that the culture results are not an infection agent, the tub is prepared for a new birth.

We tried to investigate the effect of immersion during labor on postpartum bleeding by calculating the reduction rates of the hematocrit values of the patients.

\section{Material and Method}

This retrospective study was conducted at the Delivery Department of Obstetrics of the University of Health Sciences Zekai Tahir Burak Women's Health Education \& Research Hospital between January 2017 and January 2018. Ethics committee approval was obtained for the study from our hospital (\#18/27.12.17). Study and control groups included women undergoing vaginal delivery with immersion in water during labor and women undergoing vaginal delivery. Data were collected regarding all women's age, gestation, parity, birth weight, first hematocrit levels upon admission to the labor ward, and postpartum hematocrit levels checked after six hours following delivery. Exclusion criteria were pre-existing hypertension, pre-eclampsia, pre-existing diabetes mellitus, glucose intolerance, chronic diseases, premature rupture of fetal membranes and other gestational disorders. Patients who have undergone an episiotomy procedure, patients who have experienced vaginal lacerations and patients who needed additional oxytocin infusion, methyl ergovine administration and/or misoprostol utilization and primiparous patient were not included in the study due to limiting bias and directly measuring postpartum hematocrit fall without any other risk factors for postpartum hemorrhage. All data were taken from patients who have experienced uneventful spontaneous vaginal delivery. Liberally, 20 units of oxytocin were administered to all patients after delivery. The study was approved by the local ethics committee of our hospital.

Statistical analysis was performed by using IBM SPSS Statistics Software (22.0, SPSS Inc., Chicago, IL). Data has been evaluated for normal distribution by using the Kolmogorov-Smirnov test. The continuous variables were presented by means \pm standard deviation and compared by using the independent samples $t$-test when the distribution was normal. The nonparametric variables and data without normal distribution were tested by using the Mann- Whitney U test. The comparison of categorical variables was made by using Fisher's exact test, or the chi-square test according to the relevant statistical test based on patient numbers regarding compared variables. All $p$ values $<0.05$ were considered statistically significant.

\section{Results}

The study groups consisted of 84 women undergoing vaginal delivery with immersion in water during labor (group 1) and the control group (group 2) of 84 women undergoing normal vaginal delivery at the hospital. The women in the two groups were matched with respect to age, parity, birth weight and gestational age. Age, parity, gestational age in weeks, first hemoglobin ( $\mathrm{Hb}$ ) upon admission, postpartum Hb level 6 hours after delivery, first hematocrit (Hct), postpartum Hct level 6 hours after delivery, percentage of Hct reduction, and birth weight in grams among study and control groups have been compared. The mean age of the women was $29.8 \pm 4$ and $30.5 \pm 4.9$ respectively. The mean gestational age was $39.1 \pm 1.1$ and $39.4 \pm 1.2$. The mean parity was found as $2.3 \pm 0.4$ and $2.4 \pm 0.6$. The mean hematocrit difference in the first group was $2.08 \pm 1.88$ and in the second group was $3.81 \pm 1.55$. The mean percentage of hematocrit reduction in the first group was $5.71 \%$ and in the second group $10.23 \%$ (Table 1). 
Table 1: Demographic clinical and laboratory characteristics of the study group $(n=168)$

\begin{tabular}{lccc}
\hline Parameter & Water immersion $(\mathrm{n}=84)$ & Control group $(\mathrm{n}=84)$ & $p$ value \\
\hline Age & $29.8 \pm 4.8$ & $30.1 \pm 4.9$ & $0.39^{*}$ \\
Parity & $2.3 \pm 0.4$ & $2.4 \pm 0.6$ & $0.45^{*}$ \\
Gestational age (weeks) & $39.1 \pm 1.1$ & $39.4 \pm 1.2$ & $0.07^{*}$ \\
First Hb (g/dL) & $13.2 \pm 1.6$ & $11.8 \pm 1.2$ & $0.50^{*}$ \\
Last Hb (g/dL) & $11.1 \pm 1.5$ & $10.7 \pm 1.2$ & $0.058^{*}$ \\
First Hct (\%) & $36.3 \pm 3.8$ & $37.1 \pm 3.3$ & $0.26^{*}$ \\
Last Hct (\%) & $34.2 \pm 3.9$ & $33.2 \pm 3.1$ & $0.068^{*}$ \\
Hematocrit reduction (\%) & $2.0 \pm 1.8$ & $3.8 \pm 1.5$ & $<0.001^{*}$ \\
Percentage of hematocrit reduction (\%) & $0.05 \pm 0.05$ & $0.10 \pm 0.04$ & $<0.001^{*}$ \\
Birthweight (grams) & $3378 \pm 446$ & $3286 \pm 350$ & $0.14^{* *}$ \\
\hline
\end{tabular}

*: Mann Whitney U test $p$ value, ${ }^{* *}$ : Independent samples $t$ test $p$ value, Hb: hemoglobin, Hct: hematocrit

Postpartum fever was observed in 1 patient in the first group and 2 patients in the second group. There was no significant difference between the two groups. Episiotomy infection was not observed in the patients in both groups.

\section{Discussion}

Water birth is an option for birth all over the world. Carefully managed water birth is both an attractive and lowrisk birth management for healthy pregnancies (1). ACOG has previously stated that "immersion in water during the first stage of labor may be associated with decreased pain or the use of anesthesia and a decreased labor duration." But a warning has also been defined that there are no known benefits to either mother or baby during the second stage of labor and cause for concern of serious harm (4). Warm water immersion hydrotherapy during labor provides comfort, supports relaxation, and is a safe and effective non-pharmacologic pain relief strategy that promotes physiological childbirth (2-4).

There have been many studies regarding the benefits of immersion in water during the first stage of labor like reduced pain, episiotomy and perineal trauma rates. In a Cochrane review in 2009; immersion in water during the first stage of labor did not reduce perineal trauma and episiotomy (9). On the other hand, in a study conducted in our hospital on immersion in water during the first stage of labor reduced episiotomy for both multiparous and nulliparous women (6).

Hydrotherapy during labor may promote relaxation and decrease pain without the risks caused by other treatments. In a pilot study, the psychophysiological effects of hydrotherapy on maternal anxiety and pain during labor were examined in 2001. The findings offer preliminary support for the therapeutic effects of bathing in labor for acute, short-term anxiety and pain reduction (11). In a retrospective cohort study in 2017; immersion in water during the first stage of labor reduces the pain and pain relief that your mother feels (12).

In a retrospective study in Australia in 2013; researchers found that there was no statistically significant difference in the average blood loss after birth between the two birth groups in their study (13). Our study has been conducted by matching the study and control group for measuring the exact hematocrit fall just caused by childbirth itself without any other postpartum hemorrhage risk factor. Previous studies have demonstrated that immersion in water during the birth process, maternal oxytocin secretion decreases. This decrease might result in more effective oxytocin discharge during the postpartum period and serve as a protective factor for postpartum hemorrhage (14). The duration of the $3^{\text {rd }}$ stage of labor, which is the delivery of the placenta is also significantly reduced after water births. This minimizes the amount of blood loss during this period. The lower blood loss in water births can also be explained by the hydrostatic pressure in the pool $(15,16)$.

In this study, we tried to investigate the effect of immersion during labor on postpartum bleeding by calculating the reduction rates of the hematocrit values of the patients. Mean postpartum hematocrit level fall for women undergoing normal vaginal delivery was found to be higher than women undergoing vaginal delivery with immersion in water during labor. Immersion in water during labor might be a protective measure for postpartum hemorrhage due to its positive effects on pain, labor progress and physiology. Therefore, studies involving larger series of cases may increase the reliability of our findings.

\section{Conclusion}

As a result, we found that in our own case mean postpartum hematocrit level fall for women undergoing normal vaginal delivery was higher than women undergoing vaginal delivery with immersion in water during labor. Studies involving more patients will be guided in the future.

: Acknowledgments: "None"

Conflict of Interest: "The authors reported no conflict of interest"

Funding: "none" 


\section{References}

1. Bovbjerg ML, Cheyney M, Everson C. Maternal and newborn outcomes following water birth: The Midwives Alliance of North America statistics project, 2004 to 2009 cohort. J Midwifery Womens Health. 2016;61(1):11-20.

2. Lukasse M, Rowe R, Townend J, Knight M, Hollowell J. Immersion in water for pain relief and the risk of intrapartum transfer among low risk nulliparous women: secondary analysis of the Birthplace national prospective cohort study. BMC Pregnancy Childbirth. 2014;14:60.

3. Ohlsson G, Buchhave P, Leandersson U, Nordstrom L, Rydhström H, Sjölin I. Warm tub bathing during labor: maternal and neonatal effects. Acta Obstet Gynecol Scand. 2001;80(4):311-4.

4. American College of Obstetricians and Gynecologists' Committee on Obstetric Practice. Committee Opinion No.679: Immersion in Water During Labor and Delivery. Obstet Gynecol. 2016;128(5):e231-e236.

5. Jezová D, Vigas M, Tatár P, Jurcovicová J, Palát M. Rise in plasma beta-endorphin and ACTH in response to hyperthermia in sauna. Horm Metab Res. 1985;17(12):693-4.

6. Mollamahmutoglu L, Moraloglu O, Ozyer S, Su FA, Karayalcin R, Hancerlioglu N, et al. The effects of immersion in water on labor, birth and newborn and comparison with epidural analgesia and conventional vaginal delivery. J Turk Ger Gynecol Assoc. 2012;13(1):45-9.

7. Radunovic N, Lockwood CJ, Alvarez M, Nastic D, Berkowitz RL. Beta-endorphin concentrations in fetal blood during the second half of pregnancy. Am J Obstet Gynecol. 1992;167(3):740-44.
8. Buckley SJ. Hormonal physiology of childbearing: Evidence and implications for women, babies, and maternity care. Washington, DC: Childbirth Connections Programs, National Partnership for Women \& Families; 2015;98-100.

9. Cluett ER, Burns E. Immersion in water in labor and birth. Cochrane Database Syst Rev. 2009;(2):CD000111.

10. Eckert K. Turnbull D. MacLennan A. Immersion in water in the first stage of labor: A randomized controlled trial. Birth. 2001;28(2):84-93.

11. Benfield RD, Herman J, Katz VL, Wilson SP, Davis JM. Hydrotherapy in labor. Res Nurs Health. 2001;24(1):5767.

12. Vanderlaan J. Retrospective Cohort Study of Hydrotheraphy in Labor. J Obstet Gynecol Neonatal Nurs. 2017;46(3):403-10.

13. Menakaya U, Albayati S, Vella E, Fenwick J, Angstetra D. A retrospective comparison of water birth among women deemed to be low risk in a secondary level hospital in Australia. Women Birth. 2013;26(4):114-8.

14. Eriksson M, Mattsson L, Ladfors L. Early or late bath during the first stage of labor: a randomised study of 200 women. Midwifery. 1997;13(3):146-8.

15. Otigbah CM, Dhanjal MK, Harmsworth G, Chard T. A retrospective comparison of water births and conventional vaginal deliveries. Eur J Obstet Gynecol Reprod Biol. 2000;91(1):15-20.

16. American College of Nurse-Midwives. Hydrotherapy during labor and birth. Position Statement. Silver Spring (MD): ACNM; 2014. 